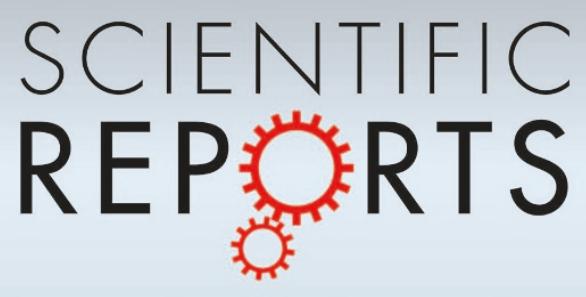

OPEN

SUBJECT AREAS:

METAMATERIALS

TRANSFORMATION OPTICS

COMPOSITES

ELECTRICAL AND ELECTRONIC ENGINEERING

Received

29 April 2013

Accepted

25 June 2013

Published

15 July 2013

Correspondence and requests for materials should be addressed to S.H. (sailing@zju.edu.

* These authors contributed equally to this work.

\section{First experimental demonstration of an isotropic electromagnetic cloak with strict conformal mapping}

\author{
Yungui Ma ${ }^{1 *}$, Yichao Liu' ${ }^{1,2 *}$, Lu Lan', Tiantian Wu', Wei Jiang', C. K. Ong ${ }^{2}$ \& Sailing He1,3
}

\begin{abstract}
${ }^{1}$ Centre for Optical and Electromagnetic Research, State Key Lab of Modern Optical Instrumentation, Zhejiang University, Hangzhou 310058, China, ${ }^{2}$ Centre for Superconducting and Magnetic Materials, Department of Physics, National University of Singapore, 2 Science Drive 3, Singapore $117542,{ }^{3}$ Department of Electromagnetic Engineering, School of Electrical Engineering, Royal Institute of Technology, S-100 44 Stockholm, Sweden.
\end{abstract}

In the past years quasi-conformal mapping has been generally used to design broadband electromagnetic cloaks. However, this technique has some inherit practical limitations such as the lateral beam shift, rendering the device visible or difficult to hide a large object. In this work we circumvent these issues by using strict conformal mapping to build the first isotropic cloak. Microwave near-field measurement shows that our device (with dielectric constant larger than unity everywhere) has a very good cloaking performance and a broad frequency response. The present dielectric approach could be technically extended to the fabrication of other conformal devices at higher frequencies.

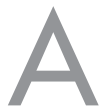

dvancement of modern transformation optics (TO) has enabled the design and demonstration of many unprecedented electromagnetic devices ${ }^{1-8}$. Among them, a successful application is to provide the concrete blueprints for invisible cloaks ${ }^{1-3}$. However, the bright future of this technique is doomed by the complexity of the transformed media that are very difficult for practical applications. For a cloaking device, the primary problem is how to effectively fulfill strong inhomogeneous anisotropic material parameters typically with sub-unity requirements. Since Pendry's first cloaking theory ${ }^{1}$ and successive experiment ${ }^{3}$, the electromagnetic (EM) cloak has experienced important advancements both in frequencies from microwave to optics ${ }^{9-12}$ and in geometries evolving from two to three dimensions ${ }^{13-17}$ excited by the modification of the original theory using quasi-conformal mapping ${ }^{18}$. However, as we know now this technique involves unequal coordinate-axis compression/extension in transformation and only produces weak anisotropic cloaking media ${ }^{19}$. One of the disadvantages is that it inherently makes a device actually detectable parallel to the lateral direction ${ }^{19,20}$. The other problem is in implementation as it is very hard to configure a device to hide large objects under the isotropic material approximation ${ }^{21}$. This can be the same reason that limits the usage of weakly anisotropic natural crystals such as calcite to achieve relatively thin cloaks compared with the invisible region, as recently demonstrated by several groups ${ }^{22-25}$, which were theoretically inspired by the bilinear general transformation ${ }^{26,27}$.

So far the quasi-conformal carpet-type cloak has been most explored in the literature for a half-space ground hiding. In 2009 Cui et al first experimentally used this technique to acquire a full-space cloak by mirroring the invisible carpet together with the metal ground along the bottom axis. In this situation an object put inside the cavity enclosed by the original and mirrored grounds was invisible for a far-field observer thus rendering a unidirectional cloaking effect ${ }^{28}$. In a later theoretical paper they proposed a line-transformed technique to have better unidirectional cloaking performance using the general transformation to avoid the inherit limits of a quasiconformal cloak but at the price of introducing anisotropic materials ${ }^{29}$. Recently Smith et al demonstrated a similar unidirectional cloaking device using homogeneous anisotropic metamaterials inspired by the bilinear transformation $^{30}$. Their device showed good cloaking performance but only for transverse electric (TE) waves and within a limited frequency band because they used magnetic resonant elements and corrugations to realize the anisotropic sub-unity permeability profile. To the best of our knowledge, so far there is still no experimental report totally solving the application issues such as the lateral beam shift, cloak size and bandwidth for carpet or unidirectional cloaking.

In this work we overcome these critical problems by first using strict conformal mapping in experiment to achieve an isotropic dielectric invisibility device and demonstrate both unidirectional and carpet-type cloaking behaviors in a wide band of microwave frequencies. Our design theory is equivalent to the one described 
in Leonhardt's 2006 paper on non-Euclidean transformation for cloaking ${ }^{2}$. However, here we only consider the first Riemann virtual sheet and reorganize the spatial geometry to obtain a unidirectional cloak for a full physical space. By adopting the half dielectric profile we fabricate a second device to demonstrate the carpet-type cloaking. Application of the conformal mapping guarantees an isotropic dielectric device without the problem of lateral beam shift ${ }^{31}$. The mapping relationship and geometrical truncation can be properly conceived to control the thickness of the final cloak. These measures help us to avoid the drawbacks encountered by the previous methods and obtain the wideband cloaking performance. The dielectric approach developed in this paper can be transferred to higher frequencies to enable the manufacture of other conformal devices by advanced fabrication technologies such as three-dimensional (3D) printing or lithography.

\section{Results}

In the reference ${ }^{2}$, conformal mapping was first introduced to achieve a full-space invisible cloak under the condition of geometrical optics where the existence of a second Riemann sheet plays a key role in rendering a hiding space. In the process the Zhukovsky transform is used to configure the coordinate projection between the virtual $(w)$ and physical $(z)$ spaces with a simple formula

$$
w=z+\frac{a^{2}}{z}
$$

where $a$ is a key parameter defining the final cloak size. The second virtual Riemann sheet is transformed into a small space with complicated material parameters which are very difficult to implement ${ }^{2}$. Here, instead of realizing a perfect cloak, we use this mapping formula to generate an isotropic line-transformed cloak by sealing off the branch-cut (entrance to the second Riemann sheet) with a metal wall as shown by the yellow line in Fig. $1 a^{32,33}$. From Eq. (1), we know this operation will lead to an invisible black hole in the transformed physical space, as shown in Fig. 1b, and the flat coordinate of the original Cartesian space in Fig. 1a will be crushed to form an infinitely large cloak shell. The red and blue lines in the figures represent light rays illuminating the sample with directions parallel and perpendicular to the metal walls (yellow lines), respectively. It is obvious that the red light will pass through the cloak and restore their directions in the far field without disturbance while the blue light will be reflected back by $180^{\circ}$ when it touches the metal wall. This conformal transformation configures an ideal unidirectional cloak with an effective hiding region of diameter equal to $a$. A far-field observer will view a piece of metal sheet (or mirror in optics) of length $=4 a$ from any off-axis angles. The material parameters of the device are
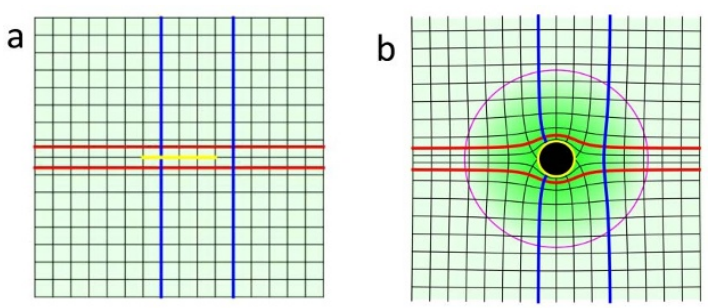

Figure 1 Conformal transformation scheme. (a) Cartesian virtual space and (b) transformed physical space. The yellow metal wall of length $4 a$ in (a) is expanded into a circle in (b) enclosing a dark invisible region of radius $a$. The red and blue lines represent two types of wave beams of orthogonal incident directions. The blue light ray hitting the metal wall will be reflected back and the others will travel forward without disturbance. The magenta dashed line in (b) describes the outer profile of the truncated cloaking device. calculated by $n=\left|\frac{d w}{d z}\right| n^{\prime}$ with $n^{\prime}$ being the refractive index in the virtual space.

Implement the unidirectional cloak. Now we go to the fabrication step. It will not be easy to manufacture such an isotropic cloaking device discussed here because the original device is infinitely large in size and has a local index distribution close to zero around the two ends at $z= \pm a$. In the cylindrical coordinate the inhomogeneous distribution of index along both radial and tangential directions is another difficult aspect in the experiment. Here we overcome these difficulties by taking three approximations: (i) The device size is suppressed by truncating the outer part where local indices are almost of no variation and the rest as a cloak device has a reasonable size compared with the cloaked region to achieve a small cloak device. Here we choose the cloak boundary (indicated by the dashed magenta circle in Fig. 1b) at radius $r=5 a$ (four times larger than the invisible hole). (ii) We assume the background of the virtual space has a moderate index $n^{\prime}=2$, and thus the region of subunity index in the transformed device is very small and can be approximated by air in the final fabricated device. (iii) The continuous cloaking model is approximated by numerous homogeneous elements with a dimension of about one tenth of local wavelength.

The validity of the above approximations is numerically examined by simulating the wave scattering for different samples as plotted in Figs. 2a to 2c. Here the incident Gaussian beam has a waist of $6 \mathrm{~cm}$ and illuminates the samples from the left side with direction parallel to the red line in Fig. 1b. The example frequency is $5.5 \mathrm{GHz}$ and the cloak parameter $a$ is equal to $3 \mathrm{~cm}$. Perfect magnetic conducting (PMC) conditions are utilized to define the exterior boundaries of the cloaked region. This selection is technically required because only a magnetic conducting line segment obeys the free-space symmetry for the excitation of the TE wave ${ }^{30}$. Figure 2 a gives the electric field pattern for the ideal case without any approximation. It shows a perfect unidirectional cloaking behavior. The reduction of the index distribution by replacing the sub-unity region with air, as shown in Fig. 2b, does slightly degrade the overall unidirectional cloaking performance with irregular side scattering. However, the good cloaking effect is basically retained. Figure $2 \mathrm{c}$ shows that the successive operations of truncation and discretization applied to the device have little effect on the unidirectional cloaking performance. These results validate our parametric and structural approximations utilized in the experiment as discussed below.

To implement the truncated device, we divided the whole $12-\mathrm{cm}$ thick cloak into 31 layers and 60 angular sectors. Each unit element has a dimension of about one tenth of the local wavelength as the permittivity $(\varepsilon)$ varies from 1 to 14.5. Rectangular metallic inclusions of different sizes have been fabricated using standard lithography of printed circuit boards (Rogers 3850) to realize the gradient index profile as shown in Fig. 3a. Dielectric powders of different dielectric constants have been used to fulfill the requirement of the wide range index. Great care has been taken to correctly fill the right powders into the right small meshes, which consumed most of the fabrication time. In order to implement the PMC boundary as required by TE polarization, here we separated the hiding region of PEC cavity from
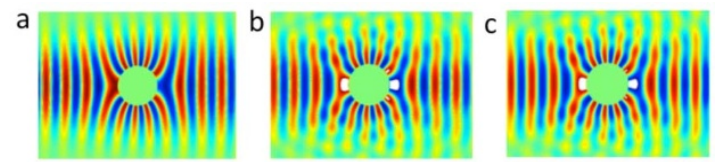

Figure $2 \mid$ Simulated cloaking behaviors. (a) The ideal case with no parametric and structural approximations; (b) the case with reduced index distribution where sub-unity regions are replaced by air; (c) the case of a truncated and discretized cloak device. PMC boundaries are used here to isolate the invisible region from the outside EM wave. 

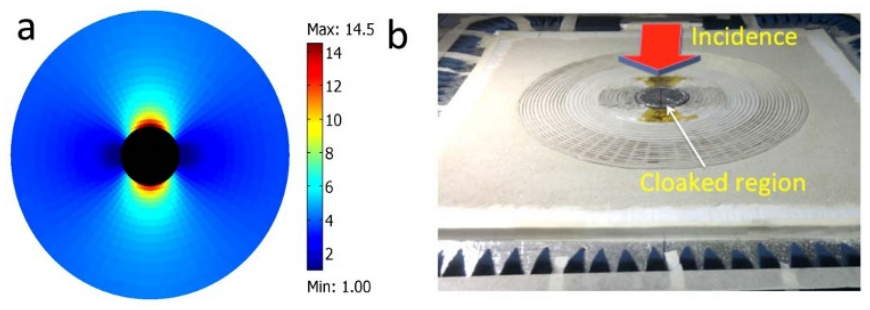

Figure 3 Unidirectional cloak. (a) Permittivity profile for the discrete device implemented here and (b) a photograph of the implemented device placed on the measurement platform. The relative permittivity has a distribution from 1 to 14.3, which changes dramatically around the invisible hole. Filling powders of different dielectric constants are used here. The white lines in (a) profile the discrete elements implemented. The red arrow in (b) represents the incident wave direction parallel to the red lines in Fig. 1b.

the cloak by a quarter wavelength dielectric doped with magnetic material (ECCOSORB ${ }^{\circledR}$ SF10 rub absorbers) ${ }^{5,34}$. The later experiment shows it has a good wideband PMC approximation. Figure $3 \mathrm{~b}$ gives a photo of our implemented device. It has a full diameter of $300 \mathrm{~mm}$ and height of $6.4 \mathrm{~mm}$. Background material consisting of powders of $\varepsilon=4$ has been used to match the impedance at the device boundary. Near the edges the background material has a linearly gradual height change trimmed from $6.4 \mathrm{~mm}$ to 0 , which could help reduce the edge scattering. The red arrow in Fig. $3 \mathrm{~b}$ represents the incident beam direction parallel to the red lines in Fig. 1b. More details about fabrication and measurement can be found in the method section.

The measured field patterns are shown in Figs. $4 \mathrm{a}$ to $4 \mathrm{~d}$ at the frequencies of 4.0, 5.0, 6.0 and 7.0 GHz. From these figures we see that the unidirectional cloaking effect is well achieved by our sample. The animation of the wave propagation at the example frequency of $5.0 \mathrm{GHz}$ is given in the supplementary Movie S1. The slightly broadening of the wave beam in Figs. $4 \mathrm{a}$ to $4 \mathrm{c}$ after passing the hiding region may be caused by two main reasons: one is the deviation of the local index from the theoretical value due to the imperfect manufacture and the other is the curved incident wavefront produced in experiment rather than the strict planar beam used in simulation. In principle our device can work for a wide frequency band.
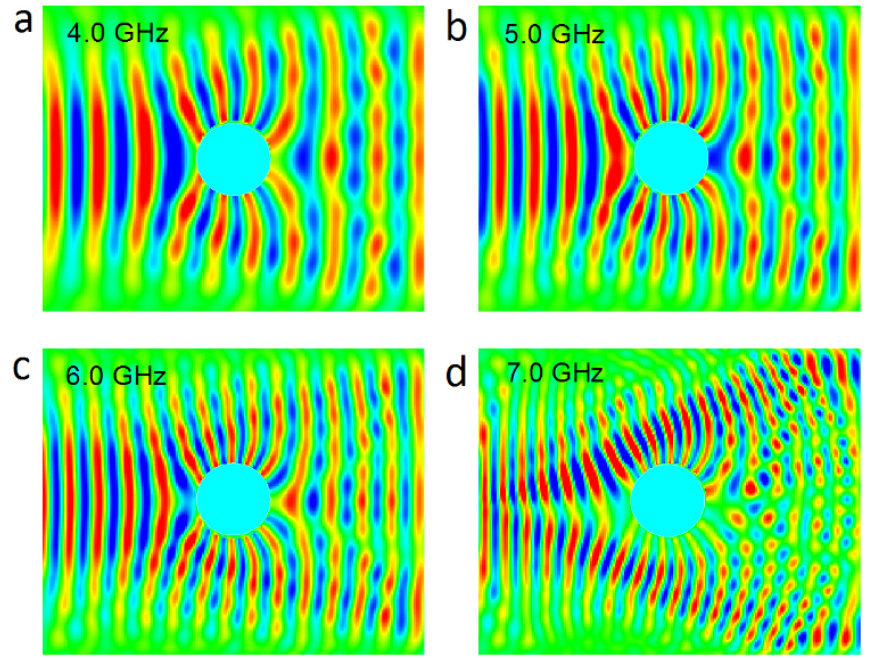

Figure $4 \mid$ Near-field measurement results for unidirectional cloak. (a), (b), (c) and (d) correspond to the measuring frequencies of 4.0, 5.0, 6.0 and 7.0 GHz, respectively. The cloaking performance is degraded at higher frequencies because of the dispersions of the metamaterial inclusions used in our samples. The cyan disk represents the hiding region.
According to the current element design, this property can be achieved for microwave frequencies up to $6 \mathrm{GHz}$ as shown by the field patterns in Figs. 4a to 4c. Beyond $6 \mathrm{GHz}$, the dispersion of the embedded metamaterial elements (metallic inclusions) becomes severe and deteriorates the cloaking performance, as manifested in Fig. $4 \mathrm{~d}$ for the frequency of $7.0 \mathrm{GHz}$. In principle the bandwidth problem could be solved by purely using natural dielectric materials to build our device.

Implement the carpet-type cloak. The carpet-type cloak predominantly discussed in the literature ${ }^{9-17,32,35-39}$ is a special case of our current design. It is fabricated by using a half dielectric profile with a PEC bottom boundary. The simulation and experimental results are given in Figs. 5 a to $5 \mathrm{f}$ at $5.5 \mathrm{GHz}$. As a comparison we first show a numerical reflection pattern of a flat mirror in Fig. 5a at the incident angle of $45^{\circ}$ for a Gaussian beam. This effect is basically reproduced by our truncated device models as shown in Figs. $5 b$ and $5 c$ for continuous and discrete media, respectively. The discretization does slightly increase the irregular scattering but is still acceptable at the working frequency below $6 \mathrm{GHz}$. The black solid lines with arrows in these figures display the wave propagation directions and the dashed lines are the extensions of these directions respectively for the incident and reflected beams in the regions outside the device. Similar to those in Fig. 5a, the two dashed lines in Figs. 5b and 5c intersect at the origin (in this case it is also the origin of the invisible half disk). These numerical results verify the predictions that the conformal device is able to solve the beam shift problem encountered by a quasi-conformal device ${ }^{19}$ and the approximations taken for implementation are reasonable. Figure $5 \mathrm{~d}$ gives a picture of our carpet-type cloak. The red arrow indicates the incident wave direction and the black line represents the metal ground. The measured field pattern shown in Fig. 5e exhibits a good mirror reflection effect and agrees with the numerical result in Fig. $5 f$ (a zoom-in figure from Fig. 5c). The animation wave pattern vividly showing the carpet cloaking is given in the supplementary Movie S2. As shown in Fig. 5e for the experimental result, the two dashed lines representing the incident and reflected wave directions intersect at a point very close to the origin. This deviation is mainly caused by the imperfect fabrication of the device and in principle could be removed by an improved fabrication. The measurement shows a similar carpet-type cloaking effect at frequencies below $6 \mathrm{GHz}$. The broadband characteristic is consistent with the previous experimental results for the unidirectional cloaking.
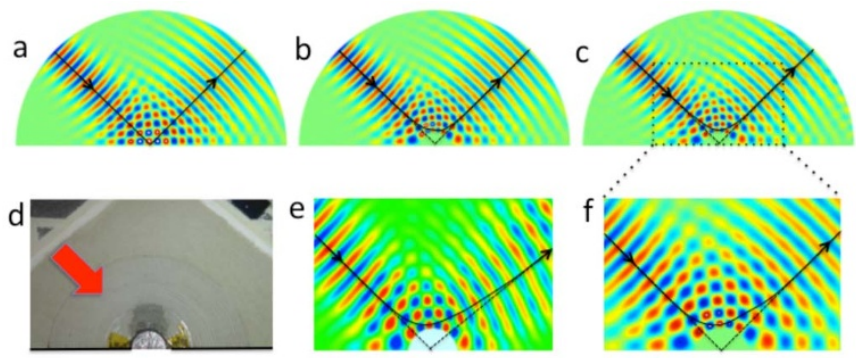

Figure $5 \mid$ Carpet-type cloak. (a) Simulated reflection from a flat mirror, (b)/(c) simulated wave scattering for our carpet device with a continuous/ discrete index profile, (d) a photo of the carpet device, and (e)/(f) measured/simulated pattern of scattered field. The black solid lines with arrows in these figures display the wave propagation directions and the dashed lines are the extensions of these directions respectively for the incident and reflected beams in the regions outside the device. Wave beam is illuminated from the top-left side at $5.5 \mathrm{GHz}$, as indicated by the red arrow in (d) which has a $45^{\circ}$ angle deviation from the normal of the yellow branch cut in Fig. 1a. Similar cloaking effect is observed at frequencies below $6 \mathrm{GHz}$. 


\section{Discussion}

We have utilized strict conformal mapping to design and fabricate an isotropic cloaking device by avoiding the drawbacks of the previous samples designed by the quasi-conformal technique. The measurement results verify the validity of our approximations made in the fabrication and elaborate good unidirectional and carpet-type cloaking behaviors. The conformal transformation helps us to obtain a large thickness ratio (0.25) of the invisible-region-to-cloak compared with the previous devices designed by the quasi-conformal method. This is easy to understand because the latter is fundamentally based on a perturbation treatment to introduce the cloaked region ${ }^{18}$. Another important result of this experiment is to show that conformal devices could be well manufactured to exhibit outstanding performance comparable with ones designed by the general TO method but with the additional advantages such as improved bandwidth and fabrication feasibility. With the help of advanced technologies such as $3 \mathrm{D}$ printing or lithography, similar conformal dielectric devices could be fabricated at THz or even optical frequencies with the index profiles carefully handled.

Here we would like to emphasize that transformation optics and the cloaking devices have already been well understood for many years and currently the key issue is how to correctly implement them. This is in line with the recently published unidirectional cloaking experiment working on TE polarization although the underlying transformation theory is well known before ${ }^{26,27}$. The previous isotropic cloaks designed by the quasi-conformal mapping have relatively small permittivity distribution (e. g., $\varepsilon=1.17$ to 2.79 for the first microwave carpet ${ }^{10}$ ) and thus are much easier for fabrication by just using patterned printed circuit boards or dielectric hole arrays ${ }^{15}$. In this work, we use a strict conformal mapping method to generate a relatively larger invisible region enabled by a larger index distribution for the cloak, i.e., $\varepsilon=1$ to 14.5 . Thanks to the different dielectric powders available (one may use e.g. Germanium for optical frequencies), we eventually managed to fabricate such a device and experimentally verified the cloaking performance. This helps to demonstrate the technical possibility of a rather complicated isotropic device. The quality of our device and the eventual performance could be enhanced by using advanced fabrication techniques as mentioned above. We believe the technical methods developed in this paper are very valuable for the development of other interesting isotropic devices designed by the conformal technique, such as the recently reported conformal lenses having illusionary optical effect ${ }^{40}$ or perfect imaging lenses ${ }^{41}$.

\section{Methods}

The simulation in this work was done by the commercial software COMSOL. The largest mesh size is smaller than one tenth of local wavelength. We use a Gaussian beam (waist $=6 \mathrm{~cm}$ ) to illuminate the device along two directions, i.e., parallel and orthogonal to the branch-cut line as shown in Fig. 1a. In the simulation and later experiment, the radius of the invisible hole is $30 \mathrm{~mm}$ and the outer radius of the cloak is $150 \mathrm{~mm}$. This corresponds to an invisible-region-to-cloak thickness ratio of 0.25 , which is a large value compared to the previous samples designed by the quasiconformal mapping. To implement the device, we divided the $120 \mathrm{~mm}$ thick cloak shell into 31 unequal layers along the radius direction and 60 sectors along the angular direction. Metamaterials made of rectangular metallic inclusions by lithography of Rogers 3850 printed circuit boards (PCBs) have been designed and used to realize the spatial index distribution. The copper cladding of this PCB has a thickness of $18 \mu \mathrm{m}$ and is patterned into differently sized rectangles to satisfy the local permittivity requirement. At a single polarization such a deep-subwavelength metal inclusion usually exhibits nearly isotropic dielectric responses as shown in the previous simulation $^{42}$. Emerson dielectric powders of epsilon $=2.5,5$ and 7 have been used together with air as background filling materials. Their density and dielectric response will approach constant after a few cycles of proper shaking. The implemented device is $300 \mathrm{~mm}$ in diameter and $6.4 \mathrm{~mm}$ in height. Along the height there are two layers of metamaterial elements. To mimic a PMC boundary separating the cloak from the invisible region, we utilized two layers of Emerson SF10 microwave absorber sheets (totally in $3 \mathrm{~mm}$ thickness) backed by an aluminum cylinder. This microwave absorber acting as a quarter wavelength spacer can reduce the electric field scattering around the inner metals. Our cloak device is embedded in a dielectric background of epsilon $=4$. We used the trimmed EPS plastic foam bars to create matched background edges by inducing a gradient height variation near each side of the rectangular background edges. Note the rigid EPS foams are transparent for microwaves and also work as pillars to support the whole sample. At about a distance of $3 \mathrm{~cm}$, we placed some microwave absorption foams to reduce the internal scattering. For the carpettype cloak, we use metal walls as the bottom ground and on the top use the embedding powders to enclose two orthogonal edges. The wave is illuminated from the top-left edge and reflected out through the top-right edge.

In the measurement, our sample was placed inside a parallel-plate waveguide chamber mounted on a platform stage driven by a $x y$-step motor. The gap width between the plates is slightly larger than the height of the sample, i.e., $6.45 \mathrm{~mm}$, where the $0.05 \mathrm{~mm}$ difference is the thickness of a plastic paper (epsilon $\sim 3$ ) covering the sample surface to protect the filling powders from friction with the top plate in the measurement. A vector network analyzer (ZVA 40) was used to generate and process the microwave signals which were guided into the measurement chamber through two coaxial cables (feeding port: a $120 \mathrm{~mm} \times 6.4 \mathrm{~mm}$ rectangular waveguide; detection port: a $2 \mathrm{~mm}$ thick monopole antenna). The detection prober is inserted into the measurement chamber through a hole drilled on the top plate and is assumed to have a distance to the sample equal to the thickness of the covering paper. Compared to the sample's thickness the influence of the $0.05 \mathrm{~mm}$ thick covering paper could be neglected. The minimum scanning step size (or the resolution of the field picture) is $1 \mathrm{~mm}$. The time interval between two points is controlled to be no less than one second (slow scanning is critically required to reduce the displacement of the loose powders).

1. Pendry, J., Schurig, D. \& Smith, D. R. Controlling Electromagnetic Fields. Science 312, 1780-1782 (2006)

2. Leonhardt, L. Optical conformal mapping. Science 312, 1777-1780 (2006).

3. Schurig, D. et al. Metamaterial electromagnetic cloak at microwave frequencies. Science 324, 977-980 (2006).

4. Leonhardt, U. \& Philbin, T. G. Transformation optics and the geometry of light. Prog. Opt. 53, 69-152 (2009).

5. Ma, Y. G., Ong, C. K., Tyc, T. \& Leonhardt, U. An omnidirectional retroreflector based on the transmutation of dielectric singularities. Nat. Mater. 8, 639-642 (2009).

6. Chen, H. Y. \& Chan, C. T. Transformation media that rotate electromagnetic fields. Appl. Phys. Lett. 90, 241105 (2007).

7. Chen, H. Y., Chan, C. T. \& Sheng, P. Transformation optics and metamaterials. Nat. Mater. 9, 387-396 (2010).

8. Xu, H., Wang, X., Yu, V., Sun, H. \& Zhang, B. Radiation-suppressed plasmonic open resonators designed by nonmagnetic transformation optics. Sci. Rep. 2, 784 (2012); DOI: 0.1038/srep00784.

9. Cai, W., Chettiar, U. K., Kildishev, A. V. \& Shalaev, V. M. Optical cloaking with metamaterials. Nat. Photon. 1, 224-227 (2007).

10. Liu, R. et al. Broadband ground-plane cloak. Science 323, 366-369 (2009).

11. Valentine, J., Li, J. S., Zentgraf, T., Bartal, G. \& Zhang, X. An optical cloak made of dielectrics. Nat. Mater. 8, 568-571 (2009).

12. Gabrielli, L. H., Cardenas, J., Poitras, C. B. \& Lipson, M. Silicon nanostructure cloak operating at optical frequencies. Nat. Photo. 3, 461-463 (2009).

13. Zhou, F. et al. Hiding a realistic object using a broadband terahertz invisibility Cloak. Sci. Rep. (2011); DOI: 10.1038/srep00078.

14. Ergin, T., Stenger, N., Brenner, P., Pendry, J. B. \& Wegener, M. Three-dimensional invisibility cloak at optical wavelengths. Science 328, 337-339 (2010).

15. Ma, H. F. \& Cui, T. J. Three-dimensional broadband ground-plane cloak made of metamaterials. Nat. Comm. 1, 124 (2011); DOI: 10.1038/ncomms1126.

16. Xu, S. et al. Experimental demonstration of a free-space cylindrical cloak without superluminal propagation. Phys. Rev. Lett. 109, 223903 (2012).

17. Wang, N., Ma, Y. G., Huang, R. F. \& Ong, C. K. Far field free-space measurement of three dimensional hole-in-Teflon invisibility cloak. Opt. Expr. 21, 5941-5948 (2013).

18. Li, J. S. \& Pendry, J. B. Hiding under the carpet: a new strategy for cloaking. Phys. Rev. Lett. 101, 203901 (2008).

19. Zhang, B. \& Wu, B.-I. Electromagnetic detection of a perfect invisibility cloak. Phys. Rev. Lett. 103, 243901 (2009).

20. Landy, N. I., Kundtz, N. \& Smith, D. R. Designing three-dimensional transformation optical media using quasiconformal coordinate transformations. Phys. Rev. Lett. 105, 193902 (2010).

21. Leonhardt, U. Towards invisibility in the visible. Nat. Mater. 8, 537-538 (2009).

22. Chen, X. Z. et al. Macroscopic invisibility cloaking of visible light. Nat. Comm. 2, 176 (2011); DOI: 10.1038/ncomms1176.

23. Zhang, B. L., Luo, Y., Liu, X. G. \& Barbastathis, G. Macroscopic invisibility cloak for visible light. Phys. Rev. Lett. 106, 033901 (2011).

24. Chen, H. \& Zheng, B. Broadband polygonal invisibility cloak for visible light. Sci. Rep. 2, 255 (2012); DOI: 10.1038/srep00255.

25. Zhang, J. J., Liu, L., Luo, Y., Zhang, S. \& Mortensen, N. A. Homogeneous optical cloak constructed with uniform layered structures. Opt. Expr. 19, 8625-8631 (2011).

26. Luo, Y. et al. A rigorous analysis of plane-transformed invisibility cloaks. IEEE Trans. Antennas. Propag. 57, 3926-3933 (2009).

27. Xi, S., Chen, H., Wu, B.-I. \& Kong, J. A. One-directional perfect cloak created with homogeneous material. IEEE Microw. Wirel. Compon. Lett. 19, 131 (2009).

28. Jiang, W. X., Ma, H. F., Chen, Q. \& Cui, T. J. A Class of Line-Transformed Cloaks with Easily Realizable Constitutive Parameters. J. Appl. Phys. 107, 034911 (2010). 
29. Ma, H. F., Jiang, W. X., Yang, X. M., Zhou, X. Y. \& Cui, T. J. Compact-sized and broadband carpet cloak and free-space cloak. Opt. Expr. 12, 19947-19959 (2009).

30. Landy, N. \& Smith, D. R. A full-parameter unidirectional metamaterial cloak for microwaves. Nat. Mater. 12, 25-28 (2013).

31. Ma, Y. G., Sun, F., Zhang, Y., Jin, Y. \& Ong, C. K. Approaches to achieve broadband transformation optical devices with transmuted singularity. J. Opt. Soc. Am. A 29, 124-129 (2012).

32. Zhang, P., Lobet, M. \& He, S. L. Carpet cloaking on a dielectric half-space. Opt. Expr. 18, 18158-18163 (2010).

33. Xu, T., Liu, Y. C., Zhang, Y., Ong, C. K. \& Ma, Y. G. Perfect invisibility cloaking by isotropic media. Phys. Rev. A 86, 043827 (2012).

34. Microwave Absorber: ECCOSORB ${ }^{\circledR}, \mathrm{Http}: / /$ www.eccosorb.com/productseccosorb-high-loss-absorbers.htm, Date of access: April 4, 2013

35. Xu, X. F. et al. Broadband invisibility cloak made of normal dielectric multilayer. Appl. Phys. Lett. 99, 154104 (2011).

36. Schmied, R., Halimeh, J. C. \& Wegener, M. Conformal carpet and grating cloaks. Opt. Expr. 18, 24361-24367 (2010).

37. Gharghi, M. et al. A carpet cloak for visible light. Nano Lett. 11, 2825-2828 (2011).

38. Ergin, T., Fischer, J. \& Wegener, M. Optical Phase Cloaking of 700 nm Light Waves in the Far Field by a Three-Dimensional Carpet Cloak. Phys. Rev. Lett. 107, 173901 (2011).

39. Zhou, F. et al. Hiding a realistic object using a broadband terahertz invisibility cloak. Sci. Rep. 1, 78 (2011); DOI: 10.1038/srep00078.

40. Chen, H. Y., Xu, Y. \& Li, H. On conformal lenses. arXiv: 1108.3496 (2011)

41. Ma, Y. G., Ong, C. K., Tyc, T. \& Leonhardt, U. Evidence for subwavelength imaging by positive refraction. New J. Phys. 13, 033016 (2011).

42. Ma, H. F. \& Cui, T. J. Three-dimensional broadband and broad-angle transformation-optics lens. Nat. Comm. 1, 124 (2010).

\section{Acknowledgments}

The authors are grateful to the partial supports from NSFCs 61271085, 60990322 and 91130004, the National High Technology Research and Development Program (863 Program) of China (No. 2012AA030402), NSF of Zhejiang Province (LY12F05005), the Program of Zhejiang Leading Team of Science and Technology Innovation, NCET, MOE SRFDP of China, the DIRP grant of Singapore (R144000304232), and Swedish VR grant (\# 621-2011-4620) and AOARD.

\section{Author contributions}

Y.G.M. conceived the ideal and Y.C.L. designed the experiment. Y.C.L., Y.G.M., L.L., T.T.W. and W.J. participated in the fabrication and measurement of the samples. Y.G.M. and S.L.H. wrote the article and C.K.O. and Y.C.L. reviewed it. S.L.H. supervised this study and finalized the manuscript.

\section{Additional information}

Supplementary information accompanies this paper at http://www.nature.com/ scientificreports

Competing financial interests: The authors declare no competing financial interests. How to cite this article: Ma, Y. et al. First experimental demonstration of an isotropic electromagnetic cloak with strict conformal mapping. Sci. Rep. 3, 2182; DOI:10.1038/ srep02182 (2013)

(c) (i) $(-)$ This work is licensed under a Creative Commons Attribution-

NonCommercial-NoDerivs 3.0 Unported license. To view a copy of this license, visit http://creativecommons.org/licenses/by-nc-nd/3.0 